\title{
The mechanism of self-recognition in humans
}

\author{
Marc Jeannerod \\ Institut des Sciences Cognitives, 67 Bd Pinel, 69675 Bron, France
}

Received 4 November 2002; accepted 11 November 2002

\begin{abstract}
Recognizing oneself as the owner of a body and the agent of actions requires specific mechanisms which have been elucidated only recently. One of these mechanisms is the monitoring of signals arising from bodily movements, i.e. the central signals which contribute to the generation of the movements and the sensory signals which arise from their execution. The congruence between these two sets of signals is a strong index for determining the experiences of ownership and agency, which are the main constituents of the experience of being an independent self. This mechanism, however, does not account from the frequent cases where an intention is generated but the corresponding action is not executed. In this paper, it is postulated that such covert actions are internally simulated by activating specific cortical networks or representations of the intended actions. This process of action simulation is also extended to the observation and the recognition of actions performed or intended by other agents. The problem of disentangling representations that pertain to self-intended actions from those that pertain to actions executed or intended by others, is a critical one for attributing actions to their respective agents. Failure to recognize one's own actions and misattribution of actions may result from pathological conditions which alter the readability of these representations.
\end{abstract}

(C) 2002 Elsevier Science B.V. All rights reserved.

Keywords: Vision; Proprioception; Movement related signals; Internal models; Action simulation; Motor system; Neuroimagery

\section{Introduction}

How do we recognize ourselves and how do we distinguish ourselves from other people? In this paper, we examine theoretical and experimental evidence for a set of mechanisms by which we become aware of our body and our actions. These mechanisms are interesting to consider, for a number of reasons. First, the ability to recognize oneself as the agent of a behavior - the sense of agency-is the way by which the self builds as an entity independent from the external world. Second, by way of consequence, self-recognition is a prerequisite for attributing a behavior to its proper agent (be it oneself or another person) and ultimately for establishing social communication with our conspecifics. One of the main outcomes of the paper will be that our body is a behaving body, and that self-recognition is largely dependent on the recognition of one's own actions. Thus, the distinction between self-generated actions and actions produced by other agents, and the corresponding ability to attribute an action to its agent will appear to be key functions for self-recognition.
The self/other distinction of the origin of an action may seem relatively simple when movements are overtly executed. In this condition, there is a possibility for monitoring sensory signals arising from the moving limbs and from the effects on the external world of the movements produced either by the self or the other, and comparing them with the signals resulting from the action generation mechanism. Which renders this distinction difficult is the existence of a number of situations where the action generation mechanism is activated, but where the action remains covert. In this paper, we will consider two such situations. One is that of imagined actions which has been extensively studied under the heading of "motor imagery"; another one is that of observation of actions performed by someone else, a situation which has been introduced more recently within the realm of covert action. Although the two situations are clearly dissimilar, they have in common to both rely on neural simulation of the imagined or the observed action.

The existence of covert action, which is an essential constituent of motor cognition, raises the problem for the self of disentangling from one another different modalities of motor representations which may be present more or less simultaneously within the same brain. Action recognition thus cannot be treated separately from the more general process

E-mail address: jeannerod@isc.cnrs.fr (M. Jeannerod). 
extending to recognition of action-related mental states like intentions or desires. In this paper, we will develop the concept of "shared representations" as a potential difficulty for action recognition and self-recognition both in normal subjects and in subjects suffering pathological conditions.

\section{Ownership and authorship in self-recognition}

In the present section, we will focus on identifying the constituents of self-recognition in adult subjects. There are several potential sources of information which may contribute to self-recognition. First, the matching of visual, tactile and proprioceptive signals originating from the same body parts contributes to an intermodal sensory image of the body. Second, the matching of one's intentions and the bodily effects of self-generated actions contributes to a sense of the self as an agent.

Everybody can experience that recognizing one's own body is not always simple, even when one sees one's face [1]. Consider for example a situation like seeing oneself in a mirror: because there is discontinuity between the body part we see and the rest of the body, an active process must take place in order to refer the body part to a representation of the whole body, what Gallagher calls our body image. According to this author [2,3], the body image is a representation (sometimes conscious, sometimes not) of an owned body, one that belongs to the experiencing self.

Sensory cues contribute to the body image. Many experimental results stress a prevalent role of vision over other senses in self-recognition: we feel our hand where we see it, not the converse. Optical distortion of the visually perceived position of a limb with respect to its felt position (e.g. by wearing laterally displacing prisms) produces no alteration of the sense of ownership: the position sense is actually recalibrated to conform with the visual information [4]. This prevalence of vision was confirmed in experiments using a rubber hand. Botvinick and Cohen [5] positioned a realistic rubber arm in front of subjects, while their real arm, hidden by a screen, was placed aside: tactile stimulation was applied simultaneously to the real and the rubber arms. After some time, the subjects experienced an illusion in which they felt the touch at the locus of the rubber arm (that they could see), not of their real (hidden) arm. In other words, the tactile stimulus was felt at the place where it was seen, at the expense of a distortion of the felt position of the real arm. In addition, subjects spontaneously reported experiencing a clear sense of ownership for the rubber arm. According to other authors who replicated this experiment, the illusion of displacement of the tactile stimulus and the illusion of ownership disappear if the rubber arm is not properly aligned with the subject's body [6]. Indeed, simply looking at a moving limb superimposed to one's own limb creates a strong impression of having willed this movement and of being its author. Observations have been reported in amputated people who experience having a phantom limb. When their valid limb is visually transposed (by way of mirrors) to the amputated side, and when they produce movements with that limb, they experience a strong feeling of voluntary movement of the phantom limb. The same happens if the visually transposed limb is that of an experimenter [7]. As argued by Wegner [8], these observations reflect a tendency to perceive oneself as causal. Even if what we experience reflects a simple (visual) appearance of mental causation, when we experience it, we tend to attribute the cause to ourselves.

Body ownership, however, is only part of the problem of self-recognition. The self is most of the time an acting self. Body parts are moving with respect to one another and with respect to external objects as the result of intentional actions. It is common experience that our actions are readily self-attributed as a consequence of a normally perfect correlation between their expected effects and the flow of resulting (visual and proprioceptive) stimulation. This matching process provides the agent of an action with the sense that he is causing that action (the sense of agency). As it is illustrated by a famous movie scene, an efficient means for determining ourselves as the owner of our body and body parts as we see them is to make them to move: if the image I see in a mirror in front of me moves when I move and the two movements are congruent, then the image I see must belong to me. Babies at a very early age seem to use this congruence criterion for attributing to themselves their body parts. Developmental studies provide evidence that self-recognition appears early in life. Infants at 5 months of age are able to discriminate their own leg movements displayed in a mirror from those of another infant, presumably by making use of a perceived contingency between their own behavior and its effects [9]. As they grow older the infants' behavior will increasingly testify to their development of a conscious self-representation. Infants of 15-20 months of age, for example, will typically resolve the task of wiping a red spot stuck on their face, when they see themselves in a mirror (see [10] for review). We will examine below experiments where this notion of congruence was measured and manipulated.

Again, however, situations may arise where this attribution becomes less than obvious. In social interactions, several people may participate in the same action and interact rapidly on the same object. Playing ball games in one example. Another one is that of two surgeons operating jointly in the same surgical theater and seeing their respective hands through a magnifying lens. In both cases, there are several moving hands visible in the scene. These hands may not appear to be directly connected to the corresponding body, as in the surgical example. Yet, these movements and the corresponding hands are correctly attributed to their authors. What is meant by these examples is that attributing to oneself both the ownership of a body part and the authorship of a movement must be based on specific mechanisms which, in everyday life, are sufficiently accurate to allow unambiguous self-recognition. Both the sense of ownership and the sense of authorship concur to self-identification: it is as essential to recognize oneself as the owner of one's body as it 
is to recognize oneself as the agent of one's actions. In this section, experimental results will be provided, which point to the main cues that normal subjects use to determine both ownership and authorship.

\subsection{The Nielsen's paradigm}

Nielsen [11] designed a paradigm which answers this query. His idea was to substitute the subject's hand with an alien hand and to present the subject with hand movements which departed from his own. The question was: at which point will the subject realize the substitution or, conversely, until when will he accept the alien hand as his own. In the experiment, subjects were requested to draw a straight line in the sagittal direction on a piece of paper (Fig. 1). In one condition, they saw their own hand doing the task; in another condition, they were shown the experimenter's hand doing the same thing at about the same time. When unbeknownst to them, the experimenter's hand drew a line that deviated from the requested direction, the subjects tended to deviate the trajectory of their own (unseen) hand in the opposite direction, so as to fulfill the instruction they had received to draw a straight line. The subjects remained unaware of this deviation: when shown their deviant performance, they tried to explain it by factors from their own, such as fatigue or inattention.

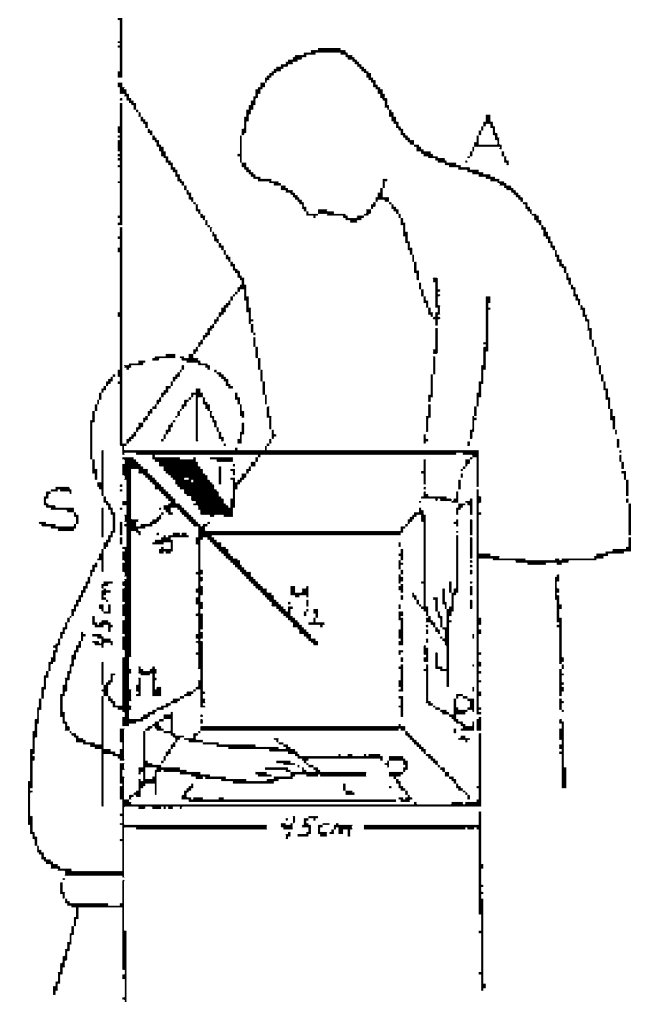

Fig. 1. Nielsen's experiment. The subject (S) looking into the box can see either his own hand or an alien hand drawing a line. The experimental situation consists in instructing the subject to draw a line and to show him the line drawn by the experimenter (from [11]).
Except for the fact that subjects were poor at recognizing their own movements and tended to misattribute to themselves movements that were not theirs, Nielsen's experiment carried no detailed information as to self-recognition. In order to specifically explore this point on a trial by trial basis, the experiment was replicated in several slightly different versions. In an experiment by Fourneret and Jeannerod [12], subjects were instructed to move a stylus in the direction of a visual target with their unseen hand: only the trajectory of the stylus was visible as a line on a computer screen, superimposed to the hand movement (Fig. 2). In some trials, a directional bias $\left(2,5\right.$ or $10^{\circ}$ to the right or to the left) was introduced electronically, such that the visible trajectory no longer corresponded to that of the hand. In order to reach the target, the hand held stylus had to be deviated in a direction opposite to the bias. In other words, although the line on the computer screen appeared to be directed to the target location, the hand movement was directed in a different direction. At the end of each trial, subjects were asked in which direction they thought their hand had moved.

This experiment revealed several important points: first, subjects accurately corrected for the bias in tracing a line which appeared visually to be directed to the target. This resulted from an automatic adjustment of their hand movements in a direction opposite to the bias. Second, subjects tended to ignore the veridical trajectory of their hand in making a conscious judgement about the direction of their hand: instead, like Nielsen's subjects, they based their report on visual cues and tended to adhere to the direction seen on the screen, thus ignoring non visual (e.g. proprioceptive) cues. The results suggest that the visuomotor system is able to appropriately use information for producing accurate corrections, but that this information cannot be accessed consciously (Fig. 3).

In another experiment using the same apparatus, the bias was progressively increased from trial to trial [13]. Although in the previous experiment the bias was randomly presented and was limited to a maximum angle of $10^{\circ}$ to the right or to the left, Slachewsky et al. used biases up to $42^{\circ}$, always presented in the same direction (e.g. to the right). When the bias amounted a mean value of about $14^{\circ}$, normal subjects changed strategy and began to use conscious monitoring of their hand movement to correct for the bias and to reach the target. In other words, the discrepancy between the seen trajectory and the felt trajectory became too large to be automatically corrected, and the failure of these corrections was compensated by conscious deviations of the hand movement in the appropriate direction. Incidentally, as shown in that same experiment, this was not true for a group of patients with frontal lesions, who apparently never became fully aware of this discrepancy, and continued to apply the automatic mechanism with the consequence of larger and larger uncorrected errors in attempting to reach the target.

The main conclusion to be drawn from these experiments is that the failure of the automatic visuomotor system (when 


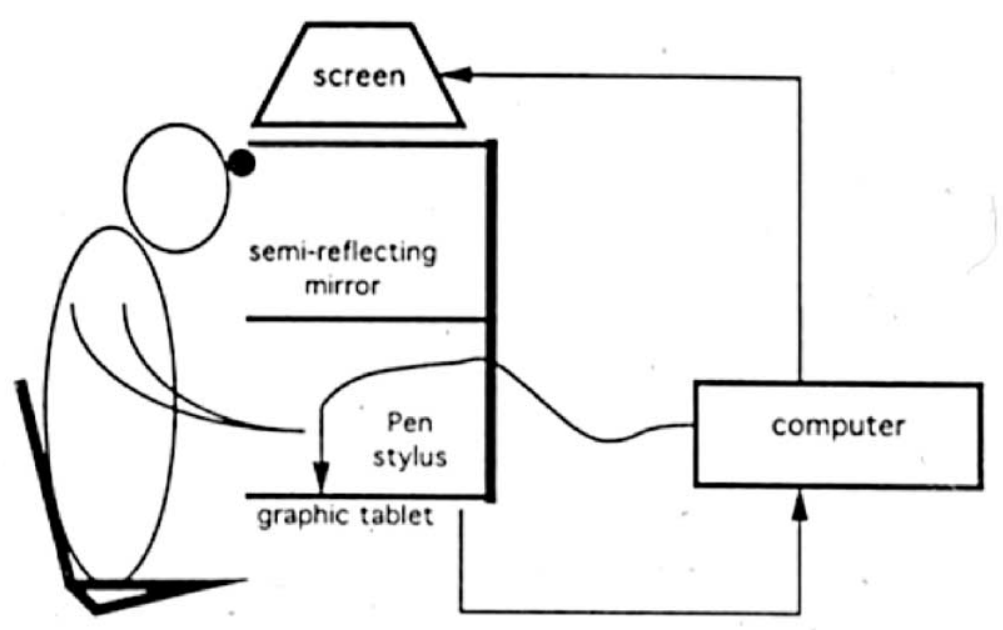

fixed seat

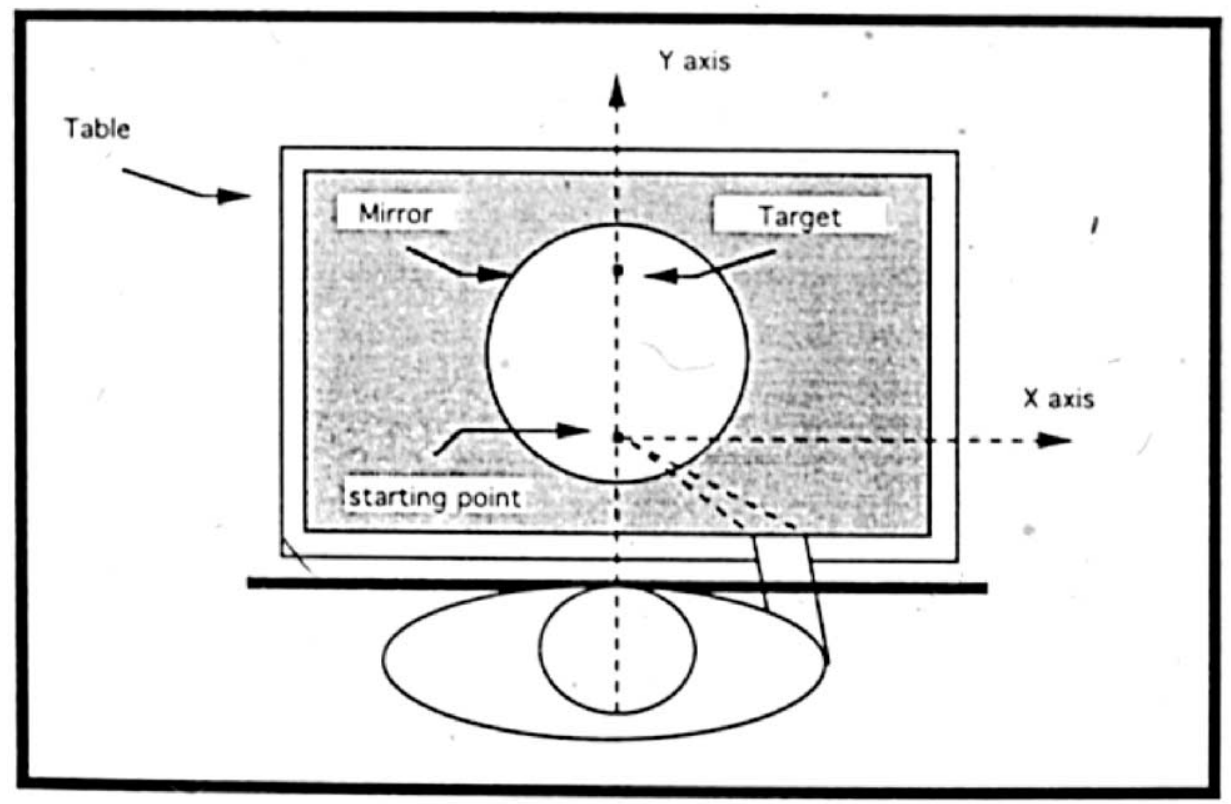

Fig. 2. Fourneret and Jeannerod's experiment. The subject using a stylus draws a line in the sagital direction on a graphic tablet. The signal is sent to a computer screen seen in a mirror covering the hand. Thus, only the line is visible. An electronic perturbation can be introduced, such that the direction of the line drawn by the subject departs from its intended direction. The subject has to move the stylus in the direction opposite to the bias in order to obtain a line in the requested direction (from [12]).

the bias becomes too large) induces a shift to a different strategy of correction for the bias, based on conscious monitoring of the error. Another conclusion is that prefrontal cortical areas are likely to be involved in this strategic shift. In a third version of the same experiment, an additional difficulty was introduced. Visual cues, which were available to the subjects in the first two experiments, were suppressed by placing an opaque mask on the screen, such that subjects could see their trajectory only in the last third of the movement. The bias was fixed at $15^{\circ}$ to the right. During the first trials, subjects made large errors with respect to the target position, which became visible when the line emerged from the mask: they had to make a correction during the last third of the movement in order to reach the target position. Normal subjects were able to progressively establish a strategy of changing the direction of their hand during the unseen part of the trajectory. Indeed, they consciously monitored their hand movement, by trying to go farther to the left. About 10 trials were needed to obtain this result. In subsequent trials, subjects were able to maintain this strategy of systematically deviating their unseen hand leftwards from the beginning of the movement, i.e. they did not have to wait for a discrepancy between the visual and the "motor" trajectories for producing a correction [14]. 

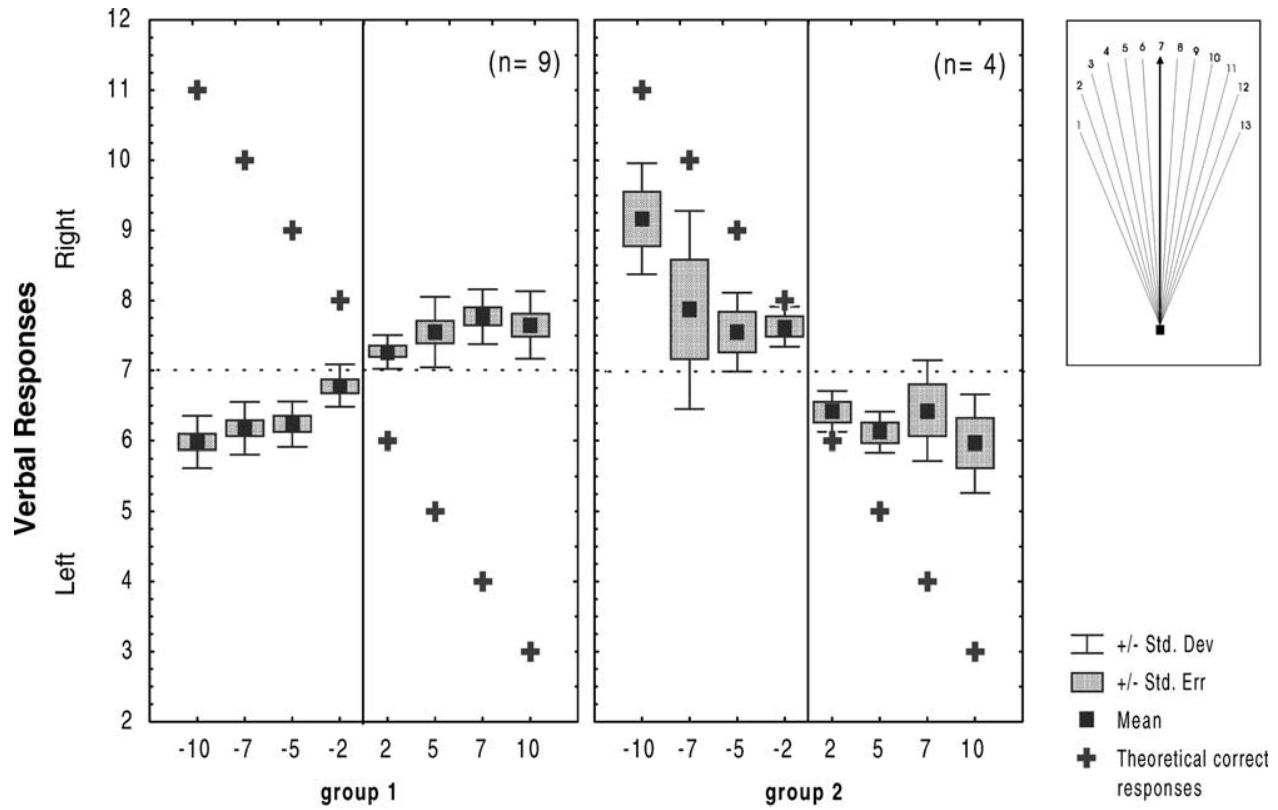

Deviation of perturbation (in degrees)

Fig. 3. Misrecognition of the direction of one's hand movements in the Fourneret and Jeannerod's experiment. The subject draws a line in the sagital direction. When a perturbation is introduced, the subject automatically deviates the hand movement in the direction opposite to the perturbation. At the end of each trial an estimate of the direction and amplitude of the hand deviation is asked to the subject. Responses are given by reading a number on the chart on the right (the number 7 corresponds to the sagital direction). Subjects tend to strongly underestimate the deviation of their hand movements. Note that subjects have been grouped according to the type of response given: subjects from group 1 tend to perceive their hand deviating in the direction opposite to the actual movement; subjects from group 2 tend to perceive their hand deviating in the veridical direction (from [12]).

Although this series of experiments dealt with recognition of action and with the degree of awareness that a subject can gain from his own movements, it said little about the cues that can be used for conscious determination of agency. This had been the objective of another set of experiments initiated by Daprati et al. [15] which explored the factors of self-attribution of a moving hand. A situation was created where the subjects were shown movements of a hand of an uncertain origin, that is, a hand that could equally likely belong to them or to someone else, also using a paradigm close to that designed by Nielsen [11]. Subjects were instructed to explicitly determine whether or not they were the author of the hand movements they saw. In order to give such a response, they had to use all available cues for comparing the current movement of their unseen hand with the movement that was displayed to them.

During the experiment, the Subject's hand and the Experimenter's hand were filmed with two different cameras. By changing the position of a switch, one or the other hand could be briefly $(5 \mathrm{~s})$ displayed on a video screen. The subject saw the screen through a mirror with an inclination of $30^{\circ}$ in the vertical plane. Subjects positioned their right hand on the table, below the mirror. The display allowed the experimenter to exactly match the image of her hand with that of the subject's hand before the beginning of each trial. Thus, looking at the mirror, the subjects got the impression that they watched their own hand. The experimenter's and the subject's hands were covered with identical gloves, in order to minimize the effects of gross morphological differences.

The task for the subjects was to perform a requested movement with their right hand, and to monitor its execution by looking at the image in the mirror. At the beginning of each experimental trial, a blank screen was presented. An instruction to perform a movement was given and the subject and the experimenter had to execute the requested movement at an acoustic signal. Once the movement was performed and the screen had returned blank, a question was asked to the subject about whether the hand that he just saw was his or not. One of three possible images of the hand could be presented to the subjects in each trial: (1) their own hand (condition: Subject), (2) the experimenter's hand performing a different movement (condition: Experimenter Different); (3) the experimenter's hand performing the same movement (condition: Experimenter Same).

Subjects were able to unambiguously determine whether the moving hand seen on the screen was theirs or not, in two conditions. First, when they saw their own hand (trials from the condition, Subject), they correctly attributed the movement to themselves. Second, when they saw the experimenter's hand performing a movement which departed from the instruction they had received (condition, Experimenter Different), they denied seeing their own hand. By contrast, their performance degraded in the condition Experimenter Same, that is, in trials where they saw the experimenter's hand performing the same movement as 
required by the instruction: in this condition, they misjudged the hand as theirs in about $30 \%$ of cases. Subjects judgement had to rely on slight differences in timing and kinematics between their intended movement and that they perceived on the screen. This result therefore indicates that the threshold for action recognition must be relatively high and that small differences tend to be neglected. This is consistent with the above observation of Fourneret and Jeannerod [12] where a small discordance between the movement and its visual consequence was ignored.

\subsection{Self-recognition in the social context}

One of the key issues of the experiments performed by Daprati and her colleagues was that, when normal subjects misrecognized the hand shown to them, they tended to overattribute that hand to themselves. One possible explanation for this effect could be that, because subjects saw only one hand and because they had moved their fingers during the presentation, the hand was automatically attributed to the author of the movements.

To avoid this possible confound, a different situation was used, which combined uncertainty about ownership of the subject's hand and uncertainty about authorship of the movements performed with that hand. This situation (see also [16-18]), involved simultaneous presentation of two hands, one of which was the subject's hand, the other being an alien hand. This situation is more realistic than the one used in previous experiments, since it involves 'social' interaction between two people, in which problems of self versus other recognition are most likely to arise. The question in this sit- uation was therefore not whether an observed action corresponded to the action one had performed, but rather which of two observed actions was the one corresponding to the action performed by the self.

The subject and the experimenter sat at the opposite sides of a table. The subject was facing an LCD screen. Both the subject and the experimenter placed their right gloved hand below the screen. A mirror attached to the backside of the screen reflected the image of the two hands to a video camera connected to a computer. A program processed the digitized video image in real time (within $20 \mathrm{~ms}$ ) and sent a black and white image of the hands onto the LCD screen. The program allowed rotating the image displayed on the screen by $-90^{\circ}$, $90^{\circ}$ and $180^{\circ}$. So, the subject could see his or her own hand at the bottom of the screen, where it would be in reality $\left(0^{\circ}\right.$ rotation), at the top of the screen $\left(180^{\circ}\right.$ rotation), at the left of the screen $\left(90^{\circ}\right.$ rotation) or at the right of the screen $\left(-90^{\circ}\right.$ rotation), while the experimenter's hand was always in the opposite direction. The rotations provided the possibility to study how the recognition of one's hand was influenced by the location where it appeared visually (Fig. 4).

At the beginning of each trial, the subject was instructed to either extend the index finger or the thumb, or to make no movement. During the trials where the subject was instructed to make a movement, the experimenter would either make the same or the alternative movement. Once the movements were performed, the screen returned dark within about $1 \mathrm{~s}$. Then a pointer was placed at the position where one of the two hands had been. Subjects had to determine whether the hand indicated by the pointer was theirs or that of the experimenter.
(A)

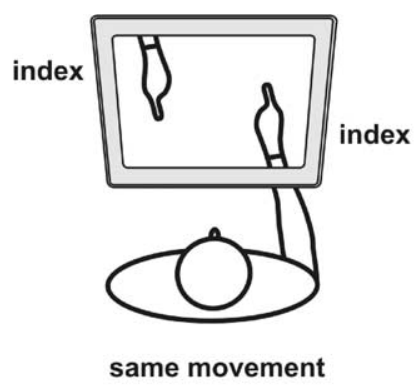

(B)

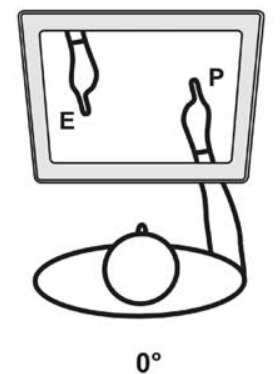

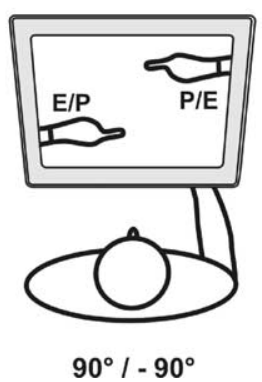

Fig. 4. Experimental conditions used in the experiment by van den Bos and Jeannerod [17]. The participant's hand (P) and the experimenter's hand (E) are shown on a screen. The two hands can appear immobile or making different movements (e.g. extend thumb or extend index), or the same movement. In addition, the hands may appear in their correct orientation with respect to the participant's body, or rotated by a variable amount. At the end of each trial the subject is instructed to attribute one of the two hands to its owner (from [18], with permission). 
This experiment first allowed to study the role of the apparent positions of the hands on self-recognition. When the two hands visually appeared at the loci corresponding to their real positions, subjects showed relatively little difficulty in recognizing their own hand. However, when the apparent locations of the hands were interchanged with respect to reality, they made attribution errors. This confirms that the contingency between visual and proprioceptive signals plays a role in self-recognition.

The most critical factor for correct attribution was the presence of finger movements. When finger movements were present and these movements were clearly attributable to the self (i.e. they differed from those of the experimenter), no attribution errors were found. This result replicates the Daprati et al.'s [15] findings, where subjects correctly attributed the hand they saw when the finger movements were theirs or when the hand was that of the experimenter performing different movements. The surprising finding in the present experiment is that accurate self-recognition was possible for all orientations of the display, including the $180^{\circ}$ rotation. In other words, when distinctive movements are available, subjects tend to recognize actions, not just hands. By contrast, when the two hands performed the same movements or no movements, the error rate increased as a function of the degree of rotation. Finally, the direction of errors in this experiment was important to consider: when movements were not discriminative (e.g. when they were the same or absent), subjects misattributed the indicated hand more often to themselves than to the other (Fig. 5). Thus, the above three experiments using the Nielsen paradigm reveal that in conditions where signals for self-recognition are weak, subjects tend to overattribute the movements to themselves. This finding has important implications for understanding the pattern of misattribution in pathological conditions that will be mentioned at the end of the paper.

\section{The nature of the mechanisms for self-recognition and attribution}

There are several ways of conceiving the mechanisms involved in self-recognition. As we shall see in this section, however, most, if not all hypotheses about these mechanisms refer to one aspect of self-recognition, namely, action recognition. This predominance of action recognition over other possible cues for self-recognition, which was clearly highlighted by the above experiments, opens a number of empirical and theoretical questions.
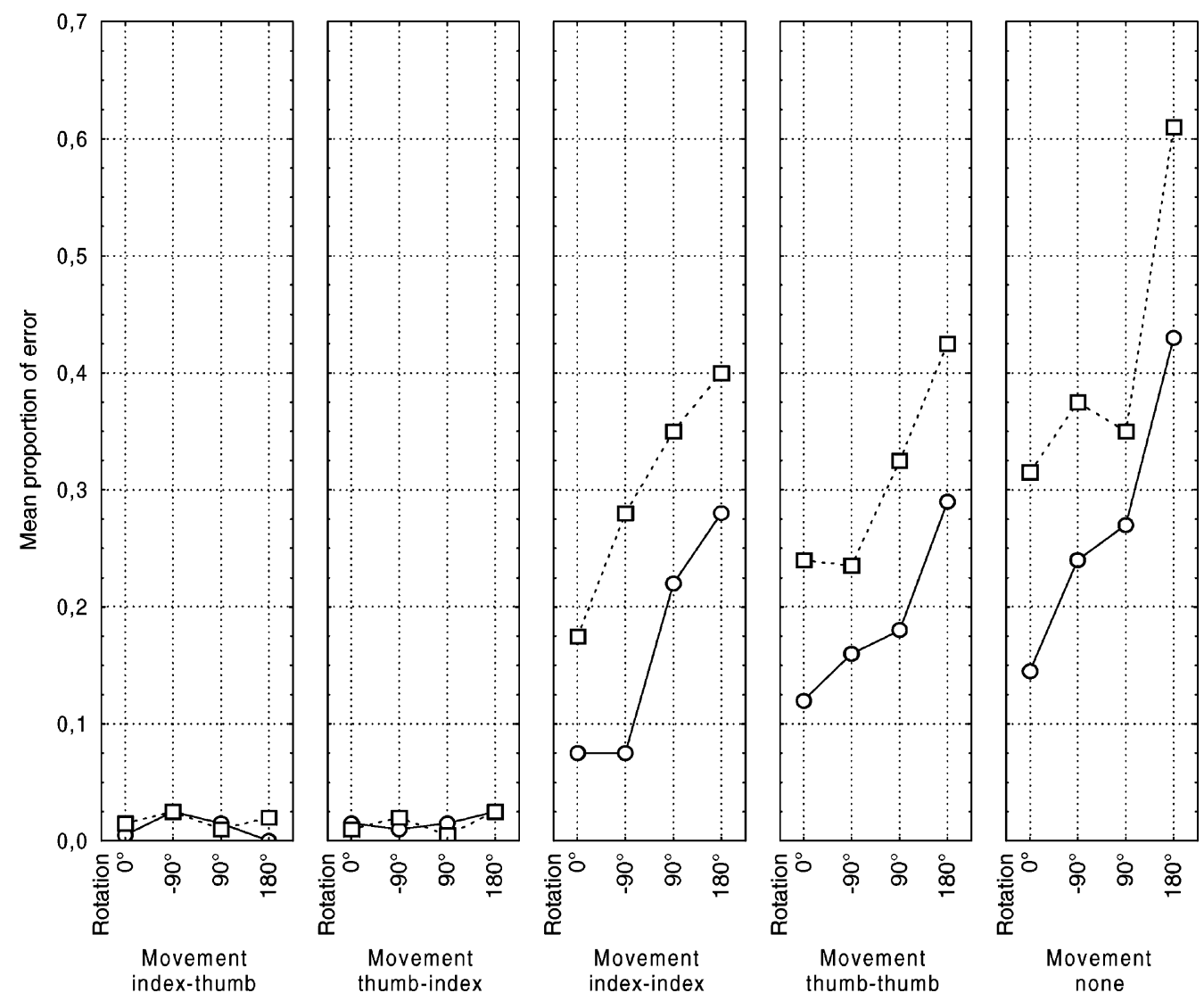
-o- Miss-attribution to other
a- Miss-attribution to self

Fig. 5. Pattern of attribution errors in the van den Bos and Jeannerod experiment. When the two hands made different movements, no attribution errors were observed, whatever the orientation of the hands. In the conditions where the movements were the same or absent, errors appeared, which were a function of the degree of rotation of the hands. Note more frequent errors by overattribution to the self (from [17]). 
In the following paragraphs, two empirically-based hypotheses of action recognition will be examined. The first hypothesis relies on the idea that executed actions generate signals which are centrally monitored and compared: action recognition arises as the outcome of this comparison (the central monitoring hypothesis). The other hypothesis relies on the idea that actions, whether or not they come to execution, are centrally simulated by the neural network, and that this simulation is the basis for action recognition and attribution (the simulation hypothesis). These two theories will appear to be largely complementary.

\subsection{The central monitoring hypothesis of action recognition}

The first hypothesis to be considered, that which holds that the comparison between efferent signals at the origin of an action and those which arise from its execution (the reafferent signals) provides cues about where the action originates, is deeply rooted in physiological thinking. Let us first rephrase its basic principles. The original idea, inherited from the cybernetic era and still operational nowadays, is that each time the motor centers generate an outflow signal for producing a movement, a copy of this command (the "efference copy") is retained. The reafferent inflow signals generated by the movement (e.g. visual, proprioceptive) are compared with the copy. If a mismatch arises between the two types of signals, new commands are generated until the actual outcome of the movement corresponds to the desired movement [19,20].

An essential aspect of this mechanism is its predictive nature. It is based on "internal models" where the states of the motor system are represented: the current state of the system, its desired state and its predicted state. Within the internal model, predictors capture the causal relationships between actions and their consequences, whereas controllers provide the necessary motor commands. Prediction in a sensorimotor system is needed to evaluate sensory information resulting from a movement. The sensory consequences of a self-produced movement can be accurately predicted from the efference copy generated in parallel with the motor command: there should normally be little discrepancy between the predicted and the actual sensory feedback. In contrast, sensations arising from the outside cannot be predicted and will result in a higher degree of discrepancy. The same stimulus will therefore be perceived differently whether it arises from a self-produced movement or it is externally produced (see [21,22]).

This model of the control of action can be directly applied to the problem of self-recognition. Self-recognition can be based on the concordance between a desired (or intended) action and its sensory consequences, which can be used to assess attribution of action to the self. This hypothesis can be tested experimentally. Haggard et al. [23], using a paradigm initiated by Libet et al. [24], have instructed subjects to make a simple voluntary movement (a key press) at a time of their choice. The action of pressing the key caused an auditory signal to appear after a fixed delay of $250 \mathrm{~ms}$. In separate sessions, the subjects were asked, either to report the position of a clock hand at the time they thought they had pressed the key, or at the time where they heard the auditory signal. Haggard et al. found that the time interval between the two estimated events was shorter than what it should be, i.e. $250 \mathrm{~ms}$. Subjects tended to perceive their key press occurring later, and the auditory signal occurring earlier, than it was actually the case. This shrinkage of perceived time between the two events did not happen in a control situation where the finger movement was not voluntary, but was produced by a magnetically induced stimulation of motor cortex. The authors conclude that intentional action binds together the conscious representation of the action and its sensory consequences. This binding effect would thus account for the self-attribution of their own actions shown by normal subjects.

A further step in identifying this mechanism is to compare brain activity during the processing of externally produced stimuli and stimuli resulting from self-produced movements. Blakemore et al. [25] using PET found that the presentation of externally-produced tones resulted in an activity in the right temporal lobe greater than when the tones were the consequence of self-produced movements. This result suggests that, in the self-produced condition, the sensory signals reaching the recipient cortical area in the temporal lobe would be modulated by the central command signals originating from the volitional system. This mechanism thus represents a powerful means of determining whether a sensory event is produced by one's own action or by an external agent (and ultimately, if an action is self-produced or not). Another PET experiment [26] explored the effect of a conflict between finger movements executed by a subject and the visual feedback given to the subject about his movements. When the executed finger movements no longer correlated with the seen ones, an increased activity was observed in the posterior parietal cortex (areas 40 and 7) bilaterally. Activation of dorsolateral prefrontal cortex on both sides was also found.

A new experiment using a similar paradigm was undertaken by Farrer et al. [27]. In this study, it was conjectured that processes underlying the sense of agency or the consciousness of action should not be all or none states, but should rather be based on continuous monitoring of the different action-related signals, from sensory (kinesthetic, visual) and central (motor command) origin. To test this hypothesis, Farrer et al. devised an experimental situation where the visual feedback provided to the subjects about their own movements could be either congruent or distorted to a variable degree. The degree of distortion went up to the point where the seen movements were completely unrelated to the executed ones. Thus, in the congruent condition, the subjects were likely to feel in full control of their own movements, whereas in the maximally distorted condition, they were likely to feel that they were not in control, 
but rather being overridden by the movements of another agent. The subjects were instructed to continuously move a joystick with their right hand. The hand and the joystick were hidden from subjects' view. Instead, the subjects saw the electronically reconstructed image of a hand holding a joystick appearing at the precise location of their own hand. When the subject moved, the electronic hand also moved by the same amount and in the same direction: subjects rapidly became acquainted to this situation and felt the movements of the electronic hand as their own. Distortions were introduced in this system, such that the movements seen by the subjects could be rotated with respect to those they actually performed (this situation was designed by Franck et al. [28]). A graded rotation was produced by using a $25^{\circ}$ rotation, a $50^{\circ}$ rotation and finally a situation where the movements appearing on the screen had no relation with those of the subjects (they were actually produced by an experimenter). Subjects were instructed to concentrate on their own feelings of whether they felt in control of the movements they saw.

Introducing a discordance between executed movements and the visual reafference from these movements produced an activation in several brain areas: the rostral part of the dorsal premotor cortex, the pre-SMA, and the right anterior cingulate gyrus were involved. The most interesting result, however, was an activation at the level of the inferior parietal lobule on the right side (Fig. 6). In concordance with Fink et al. [26], the results of this experiment demonstrate that a decreasing feeling of control due to larger and larger degrees of distortion was associated with increased $\mathrm{rCBF}$ (measured with PET) in the right inferior parietal lobule and, to a lesser extent in a symmetrical zone on the left side. Interestingly, the peak activation in the right inferior parietal lobule (in area 39) was modulated as a function of the feeling experienced by the subject of being in control of the action. The graded activation of the right inferior parietal lobe thus related to the increased degree of discordance between central signals arising from the motor command and visual and kinesthetic signals arising from movement execution. The mismatch between normally congruent sets of movement related signals is likely to result in an increased level of processing of these signals. The role of the right inferior parietal lobule, which is demonstrated by the above experiments [26,27] is consistent with the effects of lesions in this area. Patients suffering from such lesions frequently deny ownership of the left side of their body. They may even report delusions about their left body half by contending that it belongs to another person in spite of contradictory evidence from touch or sight [29,30]. Conversely, a transient hyperactivity of a similar area of the parietal lobe (during epileptic fits for example) may produce impressions of an alien phantom limb (see [31]). Finally, Sirigu et al. [32], using the same paradigm as Daprati et al. [15] found that parietal lesioned patients fail to discriminate their own actions from actions performed by an experimenter. These observations stress the role of the parietal lobe in integrating these signals for building representations essential for self-recognition.
The problem raised by these results is to determine how the modulation of activity for different degrees of discordance between an intended and an executed action, mainly in the posterior parietal cortex and in the dorsolateral prefrontal cortex, can be at the origin of the feelings of being in control of an action and attributing an action to oneself. The action monitoring model capitalizes on peripheral signals produced by the subject's motor activity. It postulates that these signals are used for comparison with the internal model of the action. There are many situations, however, where an action representation is formed but no movement is executed. In such situations, no output signals to the muscles, no reafferent (e.g. visual) signals from the outside world, no proprioceptive signals (and therefore, no possibility for comparing execution with a desired output) exist. Yet, the attribution of the representation is clearly made to the self.

\subsection{The simulation theory: from motor imagery to action attribution}

The notion that a movement should be executed for the self-recognition to operate is a problem in itself. As already stated in Section 1, action often remains within the mind without becoming apparent. The existence of overt behavior is not a prerequisite for self-attribution. It has been often argued that thinking, which normal subjects unambiguously attribute to themselves, is an equivalent of a weak form of behavior which does not activate muscles and is therefore invisible from the outside (see $[8,33,34]$ ). A paradigmatic situation where this occurs is motor imagery. In this section, we will describe recent research on motor imagery and look for another explanatory model of action recognition, attribution and self-recognition based on neural simulation of actions.

Our hypothesis thus postulates that covert actions like motor images are in fact actions on their own right, except for the fact that they are not executed. Covert and overt stages represent a continuum, such that every overtly executed action implies the existence of a covert stage, whereas a covert action does not necessarily turns out into an overt action. Most of the neural events which lead to an overt action already seem to be present in the covert stages of that action. Being provocative, one might tentatively propose that a covert action includes everything that is involved in an overt action, except for the muscular contractions and the joint rotations. Even though this contention is factually incorrect, as we know that the musculo-articular events associated with a real movement generate a flow of (reafferent) signals which are not present as such in a covert action, it captures the functioning of the representation. The theory therefore predicts an identity, in neural terms, of the state where an action is simulated and the state which immediately precedes execution of that action [35].

Interest in the field of mental imagery stemmed more than a century ago in the context of hypnosis. To explain hypnotic 


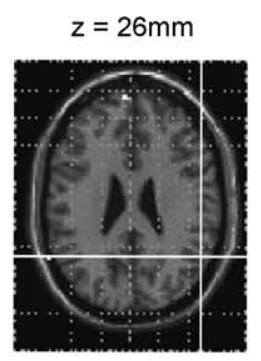

$z=34 \mathrm{~mm}$

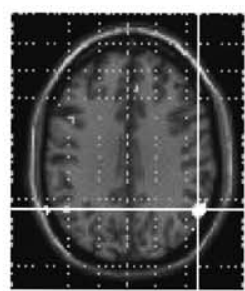

$\mathrm{z}=40 \mathrm{~mm}$

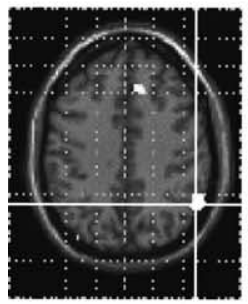

$z=46 \mathrm{~mm}$

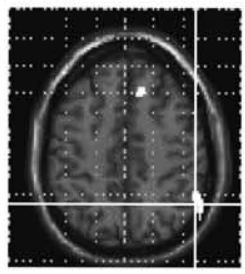

$z=52 \mathrm{~mm}$

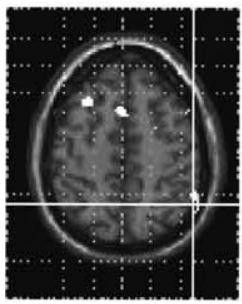

$z=28 \mathrm{~mm}$

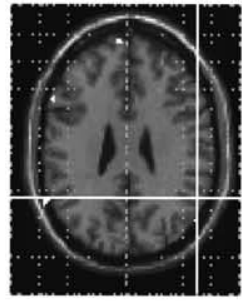

$z=36 \mathrm{~mm}$

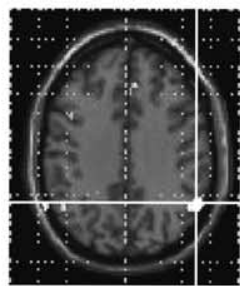

$z=42 \mathrm{~mm}$

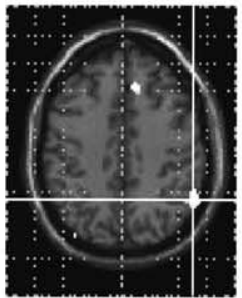

$z=48 \mathrm{~mm}$

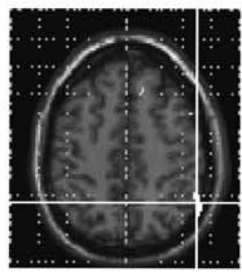

$z=54 \mathrm{~mm}$

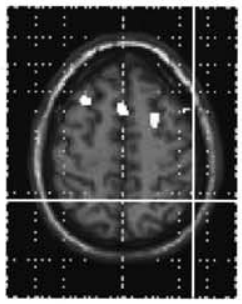

$z=30 \mathrm{~mm}$

T value

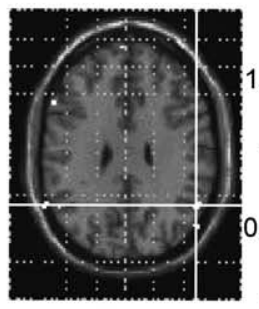

$z=38 \mathrm{~mm}$

$T$ value

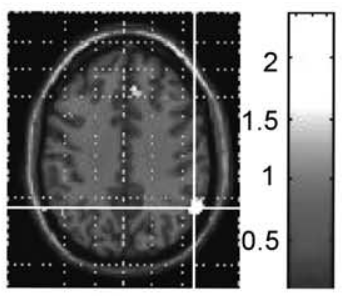

$z=44 \mathrm{~mm}$

T value

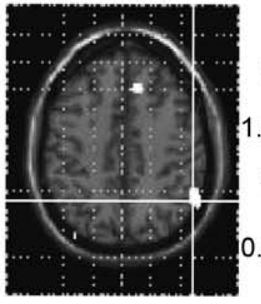

$z=50 \mathrm{~mm}$

T value

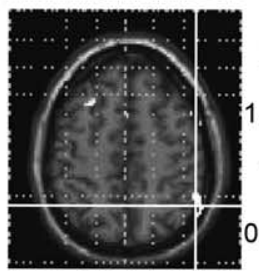

$z=56 \mathrm{~mm}$

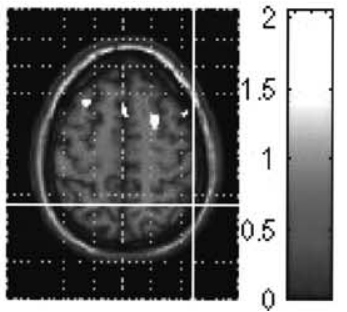

Fig. 6. Activation of the right posterior parietal lobe in a self/other conflicting situation. In a PET experiment by Farrer et al., subjects were shown hand movements which did not correspond to the movements they were actually performing. This conflict between intended movements and their resulting visual feedback activated an area located in Brodman areas 39 and 40, predominantly on the right side. The degree of activation of this area increased as a function of the amount of conflict: maximal activation occurred when the movements shown to the subject were unrelated to his own movements (from Farrer et al., in press).

phenomena, authors of the time (e.g. Binet [36]) claimed that mental images in general resulted from excitation of the same cerebral centers as the corresponding actual sensation. In the domain of motor images, it was remarked that the state of the motor centers influence the possibility to generate a motor image: for example, it was shown to be impossible for a subject to generate the image of pronouncing the letter /b/ if he kept the mouth wide open: this was because, supposedly, the motor system cannot be engaged in two contradictory actions at the same time. More recently, imagined actions or motor images have become a major tool for the study of representational aspects of action. Among the most impressive behavioral findings revealed by motor imagery studies is the fact that motor images retain the same temporal characteristics as the corresponding real action when it comes to execution. For example, it takes the same time to 
walk mentally to a prespecified target as it takes to actually walk to the same place [37]. Similarly, temporal regularities which are observed in executed actions are retained in their covert counterparts [38]. Along the same line, other situations have been described where the subject is requested to make a perceptually-based "motor" decision. Those are situations where no conscious image is formed. Consider for example the situation where a subject is simply requested to make an estimate about the feasibility of an action, e.g. to determine the feasibility of grasping an object placed at different orientations: the time to give the response is a function of the object's orientation, suggesting that the arm has to be mentally moved to an appropriate position before the response can be given. Indeed, the time to make this estimate is closely similar to the time it takes to actually reach and grasp an object placed at the same orientation ([39] see also [40]).

Research on motor imagery has thus reinforced the notion of action simulation, according to which covert actions are mentally (and neurally, see below) simulated by the potential agent. Hence the above hypothesis that a covert action should present the same features as a real action. The other form of covert action that has been earlier mentioned in this paper, namely observation of action performed by another person, should also be liable to the same explanation. Indeed, it has often been claimed that we understand the mind of others by simulating them, an idea which was already present in the literature a century ago under the concept of empathy [41]. Empathy expresses the possibility that we understand other people's behavior (e.g. their actions, their facial expressions, etc.) because we attempt to replicate and simulate their mental activity. In other words, the observed action would activate, in the observer's brain, the same mechanisms that would be activated, were that action intended or imagined by the observer (e.g. [42]).

Recent experiments have confirmed that action simulation can be a robust cue for recognizing both one's own actions and those observed from other people. Knoblich and Flach [43] presented to subjects videos of an action (throwing darts) that they had previously performed and videos of the same action performed by other subjects. The subjects task consisted in predicting the accuracy of the observed actions. Prediction was more accurate when subjects observed their own actions than when they observed another person's actions. This result suggests that the observation of self-generated actions is more informative, because the pattern of the observed action was produced by the same mechanism that simulates the action during the observation (see [44]).

A critical condition for assigning motor images and observed actions the status of simulated actions is that they should activate areas in the brain known to be devoted to executing actions [45]. The study of brain activity during covert actions has confirmed this prediction. Early work by Ingvar and Philipsson [46] using measurement of local cerebral blood flow with 133-Xenon had shown that "pure motor ideation" (e.g. thinking of rhythmic clenching movements) produced a marked frontal activation and a more limited activation in the Rolandic area. Later on Roland et al. [47], also found motor activation during imagined movements. Recent brain mapping experiments using PET or fMRI have lead to the conclusion that represented actions involve a subliminal activation of the motor system (see reviews in $[45,48]$ ). These studies show the existence of a cortical and subcortical network activated during both motor imagery and action observation. This network involves structures directly concerned with motor execution, such as motor cortex, dorsal and ventral premotor cortex, lateral cerebellum, basal ganglia; it also involves areas concerned with action planning, such as dorsolateral prefrontal cortex and posterior parietal cortex (see [49]). Concerning primary motor cortex itself, fMRI studies unambiguously demonstrate that pixels activated during contraction of a muscle are also activated during imagery of a movement involving the same muscle [50]. During action observation, the involvement of primary motor pathways was demonstrated using a direct measurement of corticospinal excitability by transcranial magnetic stimulation (TMS) of motor cortex [51] see also [52]. In premotor cortex and SMA, the overlap between imagined and observed actions is almost complete, as it is also in posterior parietal cortex. By contrast, action observation largely involves inferotemporal cortex, which is not the case for action imagination. Although the cortical networks pertaining to each form of covert (or represented) actions do not entirely overlap with each other, there are large cortical zones which are common to both (see [53], for review).

This relative similarity of neurophysiological mechanisms accounts for both the fact that actions can normally be attributed to their veridical author, and that action attribution remains a fragile process. Indeed, there are in everyday life ambiguous situations where the cues for the sense of agency become degraded and which obviously require a subtle mechanism for signaling the origin of an action. As the experiments above have shown, situations can be created where normal subjects fail to recognize their own actions and misattribute to themselves actions performed by another agent. This may also be the case of situations created by interactions between two or more individuals (e.g. joint attention, matched actions, or mutual imitation), or situations pertaining to the domain of man-machine interactions (e.g. telemanipulation, virtual reality systems, etc.).

\subsection{Differences and similarities between the two theories}

The simulation theory and the central monitoring hypothesis described in the previous two sections have a number of points in common. In one of its latest versions, the central monitoring hypothesis postulates that the internal model at the origin of an action can operate on a purely representational basis. According to Frith et al. [54], the internal model can estimate the current state of the motor command of a 
movement to predict the next state, by simulating the movement dynamics. It can also predict the sensory feedback that would result from the movement if it were executed. The sensory error - the difference between actual and predicted sensory feedback - can be used to correct the state estimate.

Although both theories involve some degree of simulation, however, they clearly depart from each other when the nature of the represented actions comes into consideration. As we stressed in the preceding sections, recognition of action and self-recognition are ultimately processes involving the participation of several persons, a social process. The main point is that actions of others are represented to the same extent as one's own, and because the two types of representations may be present in the same brain, they must be disentangled from each other. Self-recognition mechanisms thus cannot operate from a solipsist point of view, they must consider the existence of other agents. In the recent model, proposed by Frith et al. [54], it is suggested that self-recognition could be achieved through the interpretation of the signals related to an action: if these signals do not belong to the self, they must belong to someone else (a default attribution). In the new model that is proposed here, specific mechanisms for attribution to another agent are postulated.

In principle, a theory that accounts for correct attribution of actions and thoughts to their respective agents should predict separate neural representations for actions and thoughts of the self and the other. One specific network would subserve recognition of actions as belonging to the self. Another one should correspond to attributing actions to other persons. This prediction, however, does not seem to be fulfilled. In the above sections, the idea was developed that brain areas activated during representing self-produced actions (executed or not) and observing actions of other people partly overlap. For example, the motor brain (e.g. motor cortex, premotor cortex, etc.) appears to be activated in both cases. In other words, neural representations are shared, not only by the same structures for the two types of covert actions, but also by different brains when two persons observe their respective actions or try to understand their respective intentions: this is the concept of "shared representations", initially developed by Daprati et al. [15] and Georgieff and Jeannerod [55]. A logical consequence of this concept is that, because subjects are indeed able to disentangle the two situations, the overlapping between neural representations must be only partial. Non-overlapping zones (those areas that are activated in one condition and not in the other) would generate a specific signal for each form of representation.

This problem of specific representations for the self and the other was investigated by Ruby and Decety [56]. They instructed subjects to imagine actions from different perspectives. In the first person perspective, subjects received the instruction to imagine themselves doing the action; in the third person perspective, subjects received the instruction to imagine that they were watching somebody else doing the action. They found a clearcut difference in brain activation according to the two conditions. In the first person perspec- tive representation, a specific activation was observed in the inferior parietal lobule in the left hemisphere. By contrast, in the third person perspective representation, activation was found in a symmetrical area of the right hemisphere. Other activated areas, which were common to the two situations were also observed. Although the results of this experiment seems to provide a basis for an attribution mechanism, by assigning different areas to the self and to the other, they only partly fit the current views on the role of parietal lobes in action recognition. Patients with left sided parietal lesions, although they loose control on their own engrams and have difficulties in planning actions, which would be congruent with activation observed on that side in normal subjects when they imagine self-produced actions, also fail to recognize pantomimed actions and actions performed by others (e.g. [57]). Concerning the right side activation observed in the third person perspective, the Ruby and Decety's interpretation seems to be supported by the Farrer et al.'s data [27]. In the Farrer et al.'s experiment, the right parietal lobe was activated proportionally to the amount of discordance between the self-produced movements and their visual consequences, with a maximal activation when the subject realized that he was not the author of the movements.

\section{Conclusion. A neural hypothesis for self-recognition and its failures: the 'Who' system}

In this concluding section, a framework for integrating self-recognition to the neural substrate and for accounting for its failures is presented. Our present conception of action recognition [55,58] is based on the existence of neural networks subserving the various forms of representation of an action. Accordingly, each representation entails a cortico-subcortical network including to a various extent activation of interconnected neural structures. Although these ensembles are clearly distinct from one form of representation to another (e.g. the representation of a self-generated action versus the representation of an action observed or predicted from another agent), they partly overlap: posterior parietal and premotor areas, for example, are activated during both. When two agents socially interact with one another, this overlap creates shared representations, i.e. neural networks that are simultaneously activated in the brains of the two agents. In normal conditions, however, the existence of non-overlapping parts, as well as the existence of possible differences in intensity of activation between the activated zones, allows each agent to discriminate between representations activated from within from those activated from outside, and to disentangle that which belongs to him from that which belongs to the other. This process would thus be the basis for correctly attributing a representation (or the corresponding action) to its proper agent or, in other words, for answering the question of "Who" is the author of an action. The flow chart of Fig. 7 is a tentative illustration of the many interactions between two agents. Each agent builds in 


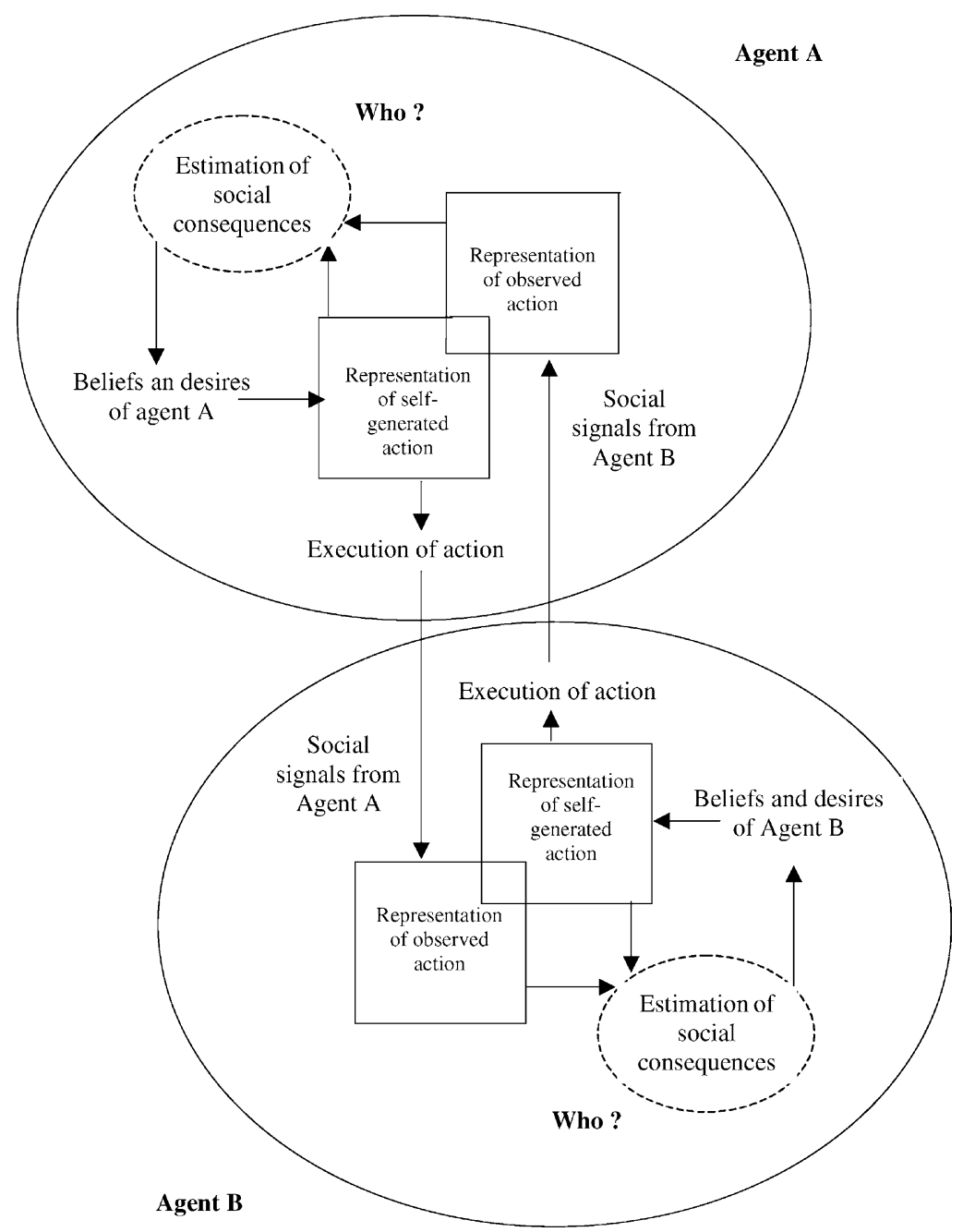

Fig. 7. Flow chart explaining the consequences of shared representations on possible misattribution of actions. The diagram depicts the interactions of two agents (A and B) observing one another. Each agent builds a representation of his own intentions/actions and of the intentions/actions of the other agent. Representations of self-generated actions and observed actions tend to overlap. An increase in overlap would render difficult attribution of these actions to their respective agent.

his brain a representation of both his own intended actions, using internal cues like his own beliefs and desires, and the potential actions of the other agent with whom he interacts. These partly overlapping representations are used by each agent to build a set of predictions and estimates about the social consequences of the represented actions, if and when they would be executed. Indeed, when an action comes to execution, it is perceived by the other agent as a set of social signals which confirm (or not) his predictions and possibly modify his beliefs and desires.

This conception allows making hypotheses about the nature of the dysfunction responsible for misattribution of actions in pathological conditions. Changes in the pattern of cortical connectivity could alter the shape of the networks corresponding to different representations, or the relative intensity of activation in the areas composing these networks. A specific configuration of symptoms appearing in schizophrenic patients has been the target of intensive search in recent years. Those are the so-called "positive" symp- toms, including verbal hallucinations, insertion of thought, and delusion of influence [59], which should represent mechanisms which normally specify the boundaries between the self and other people. Patients with positive symptoms tend to overattribute to themselves actions performed by others or, conversely, to attribute their own actions or thoughts to the influence of others. When placed in experimental situations like those already described in the previous sections, these patients were found to systematically misattribute their movements or those of the experimenter $[15,16,28,60]$.

On of the possible explanations for this failure to recognize one's own actions and to correctly attribute actions to their agents has focused on the role of prefrontal cortex. Prefrontal cortex is known to be hypoactive in many schizophrenic patients [61]. Its morphological aspect has also been shown to be modified on post-mortem examination [62]. Because prefrontal areas are known to normally exert an inhibitory control on other areas involved in various aspects of motor and sensorimotor processing, an alteration 
of this control in schizophrenic patients might result in aberrant representations for actions. Indeed, neuroimaging studies have revealed that patients presenting verbal hallucinations (e.g. "voices" whereby inner speech is erroneously attributed to external agents) show abnormal activation of primary auditory areas in their left temporal lobe, as if they were processing an external auditory stimulus [63]. Similarly, an increased activity in the right posterior parietal lobe has been observed in patients with delusion of influence, either at rest [64] or during an action recognition task [31]. Such increased activation could likely be related to the loss of frontal inhibition on the corresponding areas.

Referring to the diagram in Fig. 7, one of the two agents represented on the diagram would become "schizophrenic" if, due to an alteration in the pattern of connectivity of the corresponding networks, the degree of overlap between the representations in his brain increased in such a way that the representations would become undistinguishable from each other. The pattern of misattribution in this agent would be a direct consequence of this alteration: for example, decreased self-attribution if frontal inhibition were too strong, or increase if it were too weak.

\section{References}

[1] Kircher TTJ, Senior C, Phillips ML, Rabe-Hesketh S, Benson PJ, Bullmore ET, et al. Recognizing one's own face. Cognition 2001;78:1-15.

[2] Gallagher S. Body schema and intentionality. In: Bermudez JL, Marcel A, Eilan N, editors. The body and the self. Cambridge, MA: MIT Press; 1995. p. 225-44.

[3] Gallagher S. Philosophical conceptions of the self: implications for cognitive science. Trends Cogn Sci 2000;4:14-21.

[4] Harris CS. Perceptual adaptation to inverted, reversed and displaced vision. Psychol Rev 1965;72:419-44.

[5] Botvinick M, Cohen J. Rubber hands 'feel' touch that eyes see. Nature 1998;391:756.

[6] Farné A, Pavani F, Meneghello F, Ladavas E. Left tactile extinction following visual stimulation of a rubber hand. Brain 2000;123:3502360.

[7] Ramachandran VS, Rogers-Ramachandran D. Synaesthesia in phantom limbs induced with mirrors. Proc R Soc Lond 1996;263:377-86.

[8] Wegner D. The illusion of conscious will. Cambridge, MA: MIT Press; 2002.

[9] Bahrick LE, Watson JS. Detection of intermodal proprioceptive-visual contingency as a potential basis of self-perception in infancy. Dev Psychol 1985;21:963-73.

[10] Bahrick LE. Intermodal origins of self-perception. In: Rochat P, editor. The self in infancy. Theory and research. Amsterdam: Elsevier; 1995. p. 349-73.

[11] Nielsen TI. Volition: a new experimental approach. Scand J Psychol 1963;4:225-30

[12] Fourneret P, Jeannerod M. Limited conscious monitoring of motor performance in normal subjects. Neuropsychologia 1998;36:113340.

[13] Slachewsky A, Pillon B, Fourneret P, Pradat-Diehl P, Jeannerod M, Dubois B. Preserved adjustment but impaired awareness in a sensory-motor conflict following prefrontal lesions. J Cogn Neurosci 2001;13:332-40.

[14] Fourneret P, Franck N, Slachewsky A, Jeannerod M. Self-monitoring in schizophrenia revisited. NeuroReport 2001;12:1203-8.
[15] Daprati E, Franck N, Georgieff N, Proust J, Pacherie E, Dalery $\mathrm{J}$, Jeannerod M. Looking for the agent. An investigation into consciousness of action and self-consciousness in schizophrenic patients. Cognition 1997;65:71-86.

[16] Farrer C, Franck N, Georgieff N, Tiberghien G, Marie-Cardine M, Daléry J, et al. Confusing the self and the other. Impaired attribution of actions in patients with schizophrenia. In preparation.

[17] van den Bos E, Jeannerod M. Sense of body and sense of action both contribute to self recognition. Cognition 2002;85:177-87.

[18] Knoblich G. Self recognition: body and action. Trends Cogn Sci 2002, in press.

[19] Sperry RW. Neural basis of the spontaneous optokinetic response produced by visual inversion. J Comp Physiol Psychol 1950;43:482-9.

[20] von Holst E, Mittelstaedt H. Das Reafferenzprinzip. Wechselwirkungen zwischen Zentralnervensystem und Peripherie. Naturwissenschaften 1950;37:464-76.

[21] Wolpert DM, Ghahramani Z, Jordan MI. An internal model for sensorimotor integration. Science 1995;269:1880-2.

[22] Blakemore SJ, Wolpert D, Frith CD. Central cancellation of selfproduced tickle sensation. Nat Neurosci 1998;1:635-40.

[23] Haggard P, Clark S, Kalogeras J. Voluntary action and conscious awareness. Nat Neurosci 2002;5:282-5.

[24] Libet B, Gleason CA, Wright EW, Perl DK. Time of conscious intention to act in relation to cerebral activities (readiness potential). The unconscious initiation of a freely voluntary act. Brain 1983;102:193224.

[25] Blakemore SJ, Frith C, Wolpert D. Spatio-temporal prediction modulates the perception of self-produced stimuli. J Cogn Neurosci 1999;11:551-9.

[26] Fink GR, Marshall JC, Halligan PW, Frith CD, Driver J, Frackowiack RSJ, Dolan RJ. The neural consequences of conflict between intention and the senses. Brain 1999;122:497-512.

[27] Farrer C, Franck N, Georgieff N, Frith CD, Decety J, Jeannerod M. Modulating the experience of agency: a PET study. Neuroimage 2003 , in press.

[28] Franck N, Farrer C, Georgieff N, Marie-Cardine M, Daléry J, D'Amato T, Jeannerod M. Defective recognition of one's own actions in schizophrenic patients. Am J Psychiatr 2001;158:454-9.

[29] Bisiach E, Berti A, Dyschiria. An attempt at its systemic explanation. In: Jeannerod M, editor. Neurophysiological and neuropsychological aspects of spatial neglect. North-Holland, Amsterdam: Elsevier; 1987. p. $183-201$

[30] Daprati E, Sirigu A, Pradat-Diehl P, Franck N, Jeannerod M. Recognition of self produced movement in a case of severe neglect. Neurocase 2000;6:477-86.

[31] Spence SA, Brooks DJ, Hirsch SR, Liddle PF, Meehan J, Grasby PM. A PET study of voluntary movement in schizophrenic patients experiencing passivity phenomena (delusions of alien control). Brain 1997;120:1997-2011.

[32] Sirigu A, Daprati E, Pradat-Diehl P, Franck N, Jeannerod M. Perception of self-generated movement following left parietal lesion. Brain 1999;122:1867-74.

[33] Feinberg I. Efference copy and corollary discharge. Implications for thinking and its disorders. Schizophr Bull 1978;4:636-40.

[34] Hesslow G. Conscious thought as simulation of behavior and perception. Trends Cogn Sci 2002;6:242-7.

[35] Jeannerod M. The representing brain. Neural correlates of motor intention and imagery. Behav Brain Sci 1994;17:187-245.

[36] Binet A. La psychologie du raisonnement. Recherches expérimentales par l'hypnotisme. Alcan: Paris; 1886.

[37] Decety J, Jeannerod M, Prablanc C. The timing of mentally represented actions. Behav Brain Res 1989;34:35-42.

[38] Sirigu A, Duhamel J-R, Cohen L, Pillon B, Dubois B, Agid Y The mental representation of hand movements after parietal cortex damage. Science 1996;273:1564-8.

[39] Frak VG, Paulignan Y, Jeannerod M. Orientation of the opposition axis in mentally simulated grasping. Exp Brain Res 2001;136:120-7. 
[40] Parsons LM. Temporal and kinematic properties of motor behavior reflected in mentally simulated action. J Exp Psychol Hum Percept Perform 1994;20:709-30.

[41] Lipps T. Aesthetik: Psychologie des Schönen und der Kunst. Hamburg: Voss; 1903.

[42] Gallese V, Goldman A. Mirror neurons and the simulation theory of mind reading. Trends Cogn Sci 1998;2:493-501.

[43] Knoblich G, Flach R. Predicting the effects of actions: interactions of perception and action. Psychol Sci 2001;12:467-72.

[44] Knoblich G, Prinz W. Recognition of self-generated actions from kinematic displays of drawing. J Exp Psychol Hum Percep Perform 2001;27:456-65.

[45] Jeannerod M. Neural simulation of action: a unifying mechanism for motor cognition. Neuroimage 2001;14:S103-9.

[46] Ingvar D, Philipsson L. Distribution of the cerebral blood flow in the dominant hemisphere during motor ideation and motor performance. Ann Neurol 1977;2:230-7.

[47] Roland PE, Skinhoj E, Lassen NA, Larsen B. Different cortical areas in man in organization of voluntary movements in extrapersonal space. J Neurophysiol 1980;43:137-50.

[48] Jeannerod M, Frak VG. Mental simulation of action in human subjects. Curr Opin Neurobiol 1999;9:735-9.

[49] Gérardin E, Sirigu A, Lehéricy S, Poline J-B, Gaymard B, Marsault $\mathrm{C}$, et al. Partially overlapping neural networks for real and imagined hand movements. Cerebral Cortex 2000;10:1093-104.

[50] Roth M, Decety J, Raybaudi M, Massarelli R, Delon-Martin C, Segebarth C, et al. Possible involvement of primary-motor cortex in mentally simulated movement. A functional magnetic resonance imaging study. NeuroReport 1996;7:1280-4.

[51] Fadiga L, Fogassi L, Pavesi G, Rizzolatti G. Motor facilitation during action observation. A magnetic stimulation study. J Neurophysiol 1995;73:2608-11.

[52] Hari R, Forss N, Avikainen S, Kirveskari E, Salenius S, Rizzolatti G. Activation of human primary motor cortex during action observation: a neuromagnetic study. Proc Natl Acad Sci 1998;95: 15061-5.
[53] Grèzes J, Decéty J. Functional anatomy of execution, mental simulation, observation, and verb generation of actions: a meta-analysis. Hum Brain Mapp 2001;12(1):1-19.

[54] Frith CD, Blakemore SJ, Wolpert DM. Abnormalities in the awareness and control of action. Philos Trans R Soc Lond B Biol Sci 2000;355:1771-88.

[55] Georgieff N, Jeannerod M. Beyond consciousness of external reality. A "Who" system for consciousness of action and self-consciousness. Consciousness Cogn 1998;7:465-77.

[56] Ruby P, Decéty J. Effect of subjective perspective taking during simulation of action: a PET investigation of agency. Nat Neurosci 2001;4:546-50.

[57] Sirigu A, Cohen L, Duhamel J-R, Pillon B, Dubois B, Agid Y, et al. Congruent unilateral impairments for real and imagined hand movements. NeuroReport 1995;6:997-1001.

[58] Jeannerod M. To act or not to act: perspectives on the representation of actions. Q J Exp Psychol 1999;52A:1-29.

[59] Schneider K. Klinische Psychopathologie. Stuttgart, Thieme: Verlag; 1955.

[60] Blakemore S, Smith J, Steel R, Johnstone EC, Frith CD. The perception of self-produced sensory stimuli in patients with auditory hallucinations and passivity experiences: evidence for a breakdown in self-monitoring. Psychol Med 2000;30:1131-9.

[61] Weinberger DR, Berman KF. Prefrontal function in schizophrenia: confounds and controversies. Philos Trans R Soc B Biol Sci 1996;351:1495-503.

[62] Goldman-Rakic PS, Selemon LD. Functional and anatomical aspects of prefrontal pathology in schizophrenia. Schizophr Bull 1997;23:437-58.

[63] Dierks T, Linden DEJ, Jandl M, Formisano E, Goebel R, Lanferman $\mathrm{H}$, et al. Activation of the Heschl's gyrus during auditory hallucinations. Neuron 1999;22:615-21.

[64] Franck N, O'Leary DS, Flaum M, Hichwa RD, Andreasen NC. Cerebral blood flow changes associated with schneiderian first-rank symptoms in schizophrenia. J Psychiatr Clin Neurosci 2002;14:277_ 82. 